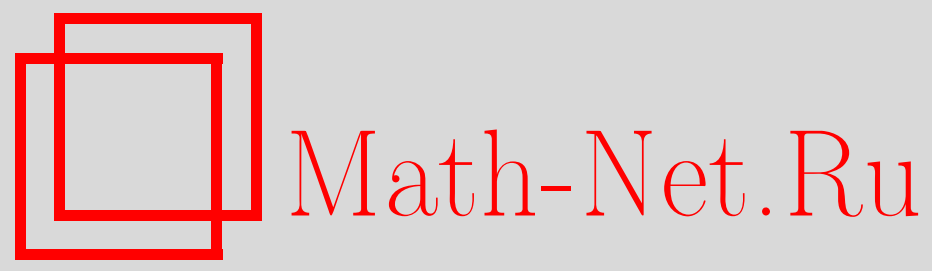

Ю. Б. Никитин, О сложности сортировки декартовых произведений частично упорядоченных множеств, Дискрет. матем., 2001, том 13, выпуск 3, 57-74

DOI: https://doi.org/10.4213/dm298

Использование Общероссийского математического портала Math-Net.Ru подразумевает, что вы прочитали и согласны с пользовательским соглашением http://www . mathnet.ru/rus/agreement

Параметры загрузки:

IP : 54.205 .225 .156

26 апреля 2023 г., 13:17:19

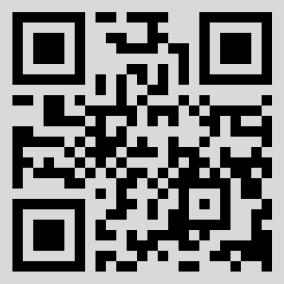


УДК 519.7

\title{
О сложности сортировки декартовых произведений частично упорядоченных множеств
}

\author{
(C) 2001 г. Ю. Б. Никитин
}

В статье изучается сложность $L\left(M_{n}\right)$ алгоритмов сортировки для частично упорядоченного множества $M_{n}$, изоморфного декартову произведению

$$
K_{1} \times \ldots \times K_{n}
$$

где все $K_{i}$ берутся из некоторого конечного семейства, имеют единственный максимальный элемент и являются простыми относительно декартова произведения. Для семейства $\left\{M_{n}\right\}$ при $n \rightarrow \infty$ установлены оценки

$$
\begin{aligned}
& L\left(M_{n}\right) \gtrsim\left|M_{n}\right| \log _{2}\left|M_{n}\right|, \\
& L\left(M_{n}\right) \lesssim\left(\left|K_{1}\right|+\ldots+\left|K_{n}\right|\right)\left|M_{n}\right| .
\end{aligned}
$$

Кроме того, для частично упорядоченных множеств с одним максимальным элементом установлен факт единственности декартова разложения на простые множители с точностью до порядка сомножителей.

\section{1. Основные определения}

Данная работа посвящена сортировке частично упорядоченных множеств. Множество $M=\left\{x_{1}, \ldots, x_{m}\right\}$ называется частично упорядоченным, если на его элементах задано отношение частичного порядка $R$. Далее мы будем использовать для частично упорядоченно множеств $M$ с отношением частичного порядка $R$ обозначение $(M, R)$. Результатом применения отношения $R$ к паре элементов $x_{i}$ и $x_{j}$ может быть одно из четырех значений: $x_{i}$ больше $x_{j}\left(x_{i}>x_{j}\right), x_{i}$ меньше $x_{j}\left(x_{i}<x_{j}\right), x_{i}$ и $x_{j}$ равны $\left(x_{i}=x_{j}\right)$ или $x_{i}$ и $x_{j}$ несравнимы $\left(x_{i} ? x_{j}\right)$. Отношение $R$ естественным образом порождает четыре отношения $>,<,=$, ? со значениями истина или ложь.

Пусть $(x \geqslant y) \equiv(x>y) \vee(x=y)$. Диаграммой Хассе частично упорядоченного множества $(M, R)$ называется ориентированный граф $G=(V, E)$, для которого существует такое взаимно однозначное отображение $f: M \rightarrow V$, что $(f(x), f(y)) \in E$ тогда и только тогда, когда

$$
|\{z:(x \geqslant z) \wedge(z \geqslant y)\}|=2 .
$$

Таким образом, выполнение отношения $x>y$ равносильно существованию в графе $G$ невырожденной ориентированной цепи из вершины $f(x)$ в вершину $f(y)$. 
Определим задачу сортировки частично упорядоченного множества следующим образом. Пусть задано множество $M=\left\{x_{1}, \ldots, x_{m}\right\}$ с отношением частичного порядка $R$ и ориентированный граф $G=(V, E)$ диаграммы Хассе частично упорядоченного множества $(M, R)$. Задача сортировки состоит в построении отображения $\varphi: M \rightarrow V$, при котором для любых $i, j \in\{1, \ldots, m\}$ соотношение $x_{i}>x_{j}$ справедливо тогда и только тогда, когда в графе $G$ существует невырожденная ориентированная цепь из вершины $\varphi\left(x_{i}\right)$ в вершину $\varphi\left(x_{j}\right)$. Далее мы будем называть отображение $\varphi$ сборкой.

Алгоритмом сортировки частично упорядоченного множества $(M, R)$ назовем произвольный алгоритм решения задачи сортировки для данного частично упорядоченно множеств, использующий результаты последовательного применения отношения $R$ к парам различных элементов из множества $M$. При этом результатом сравнения двух элементов из $M$ может быть одно из трех значений: больше, меньше или несравнимы. Время работы алгоритма сортировки при заданном входе равно количеству обращений к отношению $R$. Сложность алгоритма сортировки равна времени работы алгоритма в худшем случае (максимальному времени работы по всевозможным входам). Алгоритм, который в худшем случае делает минимальное число сравнений, мы назовем оптимальным. Сложность оптимального алгоритма сортировки множества $M$ обозначим через $L(M)$.

Описанная задача сортировки частично упорядоченного множества является обобщением задачи сортировки линейно упорядоченных множеств по убыванию в постановке $[1,2]$ (для данного линейно упорядоченного множества $L_{m}=\left\{x_{1}, \ldots, x_{m}\right\}$ с отношением порядка $>$ найти такую перестановку $\pi \in S(m)$, что $x_{\pi(i)}>x_{\pi(j)}$ тогда и только тогда, когда $i<j$ ). Особенностью задачи сортировки частично упорядоченного множества $M$ в нашей постановке является неоднозначность получаемого отображения $\varphi$ в случае существования нетривиальных автоморфизмов множества $M$. Таким образом, отображение $\varphi$ из определения задачи сортировки не всегда совпадает с отображением $f$ из определения диаграммы Хассе.

Для произвольного набора частично упорядоченных множеств $\left\{\left(M_{i}, R_{M_{i}}\right)\right\}_{i=1}^{n}$ определим декартово множество наборов как частично упорядоченное множество

$$
\left(M_{1} \times \ldots \times M_{n}, R_{M_{1} \times \ldots \times M_{n}}\right),
$$

где

$$
M_{1} \times \ldots \times M_{n} \equiv\left\{\left(x_{1}, \ldots, x_{n}\right): x_{i} \in M_{i}, \quad i \in\{1, \ldots, n\}\right\}
$$

a

$$
\left(x_{1}, \ldots, x_{n}\right) \geqslant_{M_{1} \times \ldots \times M_{n}}\left(y_{1}, \ldots, y_{n}\right)
$$

тогда и только тогда, когда $x_{i} \geqslant_{M_{i}} y_{i}$ для всех $i \in\{1, \ldots, n\}$. Остальные отношения определяются из тождеств

$$
\begin{aligned}
(x=y) & \equiv(x \geqslant y) \wedge(y \geqslant x) \\
(x>y) & \equiv(x \geqslant y) \wedge \neg(x=y), \\
(x<y) & \equiv(y>x) \\
(x ? y) & \equiv \neg((x<y) \vee(x>y) \vee(x=y))
\end{aligned}
$$

Пусть $M^{1} \equiv M$, а $M^{n} \equiv M^{n-1} \times M$ при $n \in \mathrm{N}, n \geqslant 2$. Для частично упорядоченного множества $M$ запись

$$
M \cong M_{1} \times \ldots \times M_{n}
$$


означает, что частично упорядоченное множество $M$ изоморфно декартовому множеству наборов $M_{1} \times \ldots \times M_{n}$ и называется их декартовым произведением. Если частично упорядоченное множество $M$ является декартовым произведением $M_{1} \times \ldots \times M_{n}$, то существует изоморфизм

$$
\psi: M \rightarrow M_{1} \times \ldots \times M_{n},
$$

который мы будем называть покомпонентным разложением. При этом изоморфизме элемент $x \in M$ переходит в набор $\psi(x)=\left(x_{1}, \ldots, x_{n}\right)$, где $x_{i} \in M_{i}$ при $i \in\{1, \ldots, n\}$.

Предложение 1. Пусть частично упорядоченное множество $M \cong M_{1} \times \ldots \times M_{n} u$ для каждого $i \in\{1, \ldots, n\}$ задана сборка $\varphi_{i}: M_{i} \rightarrow V_{i}$, где $V_{i}-$ множество вериин графа диаграммы Хассе частично упорядоченного множества $M_{i}$. Тогда задача сортировки частично упорядоченного множества $M$ сводится $\propto$ построению одного из покомпонентных разложений

$$
\psi: M \rightarrow M_{1} \times \ldots \times M_{n} .
$$

\section{2. Автоморфизмы декартовых произведений}

Определим функцию расстояния $\rho(x, y)$ для пар элементов $x, y$ частично упорядоченного множества $M$, полагая

$$
\begin{aligned}
\rho_{M}(x, y)=\max \left(\left\{l: x=\alpha_{0}<\alpha_{1}<\ldots<\right.\right. & \left.\alpha_{l}=y\right\} \\
& \left.\cup\left\{l: y=\beta_{0}<\beta_{1}<\ldots<\beta_{l}=x\right\} \cup\{-1\}\right) .
\end{aligned}
$$

Для частично упорядоченного множества $M$ положим

$$
\max (M)=\{x \in M: \forall y \in M((y \leqslant x) \vee(y ? x))\} .
$$

На элементах частично упорядоченного множества $M$ введем функцию ранга

$$
l_{M}(x)=\max \left\{\rho_{M}(x, y): y \in \max (M)\right\} .
$$

Функцию

$$
h(M)=\max \left\{l_{M}(x): x \in M\right\}
$$

назовем высотой частично упорядоченно множеств $M$, а множество

$$
S_{i}(M)=\left\{x \in M: l_{M}(x)=i\right\}
$$

назовем его $i$-м слоем. При этом $S_{0}(M)=\max (M)$. Положим $\min (M)=S_{h(M)}(M)$.

Предложение 2. Для пары элементов $x$ и у из иастично упорядоченного множества $M$ справедливо утверждение: если $x<y$, то $l_{M}(x)>l_{M}(y)$.

Следствие 1. Если для нехоторых элементов $x$ и у из частично упорядоченного множсства $M$ справедливо равенство $l_{M}(x)=l_{M}(y)$, то либо $x=y$, либо $x$ ? $y$.

Следствие 2. Пустъ на частично упорлдоченном множестве $M$ определен автоморяизм $\psi: M \rightarrow M$. Тогда для любого $x \in M$ справедливо равенство $l_{M}(x)=$ $l_{M}(\psi(x))$. 
Предложение 3. Пусть для частично упорядоченно множеств $M$ существует покомпонентное разложение

$$
\psi: M \rightarrow M_{1} \times \ldots \times M_{n}
$$

$u \psi(x)=\left(x_{1}, \ldots, x_{n}\right)$ для некоторого $x \in M$. Тогда

$$
l_{M}(x)=\sum_{i=1}^{n} l_{M_{i}}\left(x_{i}\right)
$$

Следствие 3. Для произвольного частично упорядоченного множества $M \cong M_{1} \times \ldots \times M_{n}$ справедливо равенство

$$
h(M)=\sum_{i=1}^{n} h\left(M_{i}\right) .
$$

Следствие 4. Если частично упорядоченное множество $M \cong M_{1} \times \ldots \times M_{n}$, то $\left|S_{0}(M)\right|=1$ mогда u только тогда, когда $\left|S_{0}\left(M_{i}\right)\right|=1$ для всех $i \in\{1, \ldots, n\}$.

Следствие 5. Если частично упорядоченное множество $M \cong M_{1} \times \ldots \times M_{n} u$ $\left|S_{0}(M)\right|=1$, mo

$$
\left|S_{1}(M)\right|=\sum_{i=1}^{n}\left|S_{1}\left(M_{i}\right)\right| \text {. }
$$

Предложение 4. Если для частично упорядоченного множества $M$ имеет место равенство $\left|S_{0}(M)\right|=1$, то граф диаграммь Хассе частично упорядоченно множеств $M$ является связным.

Пусть про декартово множество наборов $M_{1} \times \ldots \times M_{n}$ известно, что $\left|S_{0}\left(M_{i}\right)\right|=1$ для всех $i \in\{1, \ldots, n\}$. Каркасом декартова множества наборов $M_{1} \times \ldots \times M_{n}$ назовем набор его подмножеств $\left\{F_{i}\right\}_{i=1}^{n}$ такой, что

$$
\begin{aligned}
F_{i}=\left\{\left(x_{1}, \ldots, x_{n}\right) \in M_{1} \times \ldots \times M_{n}:\right. & x_{1} \in S_{0}\left(M_{1}\right), \ldots, x_{i-1} \in S_{0}\left(M_{i-1}\right), \\
& \left.x_{i} \in M_{i}, x_{i+1} \in S_{0}\left(M_{i+1}\right), \ldots, x_{n} \in S_{0}\left(M_{n}\right)\right\}
\end{aligned}
$$

для каждого $i \in\{1, \ldots, n\}$.

Предложение 5. Пусть частично упорядоченное множество $M=M_{1} \times \ldots \times M_{n}$, $\left|S_{0}(M)\right|=1$, а набор множеств $\left\{F_{i}\right\}_{i=1}^{n}$ является каркасом декартова множества наборов $M_{1} \times \ldots \times M_{n}$. Тогда $F_{i} \cong M_{i}$ для всех $i \in\{1, \ldots, n\}$.

Предложение 6. Пусть частично упорядоченное множество $M=M_{1} \times \ldots \times M_{n}$, $\left|S_{0}(M)\right|=1$, а набор множеств $\left\{F_{i}\right\}_{i=1}^{n}$ лвляется каркасом декартова множества наборов $M_{1} \times \ldots \times M_{n}$. Тогда

$$
S_{1}(M)=\bigcup_{i=1}^{n} S_{1}\left(F_{i}\right)
$$


Для частично упорядоченного множества $M$ и его подмножества $T \subseteq M$ определим множество

$$
H_{M}(T)=\{x \in M: \exists t \in T(x \leqslant t)\} .
$$

Если, кроме того, $\left|S_{0}(M)\right|=1$ и $T \subseteq S_{1}(M)$, то положим

$$
C_{M}(T)=M \backslash H_{M}\left(S_{1}(M) \backslash T\right) .
$$

Предложение 7. Для любого частично упорядоченного множества $M$ и двух его подмножеств $A, B \subseteq M$ верно, что

$$
H_{M}(A \cup B)=H_{M}(A) \cup H_{M}(B) \text {. }
$$

Лемма 1. Пусть частично упорядоченное множество $M=M_{1} \times \ldots \times M_{n}$, $\left|S_{0}(M)\right|=1$, а набор множеств $\left\{F_{i}\right\}_{i=1}^{n}$ является каркасом дехартова множества наборов $M_{1} \times \ldots \times M_{n}$. Тогда $C_{M}\left(S_{1}\left(F_{i}\right)\right)=F_{i}$ для кажсдого $i \in\{1, \ldots, n\}$.

Доказателъство. Зафиксируем $i \in\{1, \ldots, n\}$. По определению

$$
C_{M}\left(S_{1}\left(F_{i}\right)\right)=M \backslash H_{M}\left(S_{1}(M) \backslash S_{1}\left(F_{i}\right)\right) .
$$

Из предложения 6 следует, что

$$
S_{1}(M) \backslash S_{1}\left(F_{i}\right)=\bigcup_{j=1, j \neq i}^{n} S_{1}\left(F_{j}\right)
$$

Поэтому, применив предложение 7 , получаем, что

$$
H_{M}\left(S_{1}(M) \backslash S_{1}\left(F_{i}\right)\right)=H_{M}\left(\bigcup_{j=1, j \neq i}^{n} S_{1}\left(F_{j}\right)\right)=\bigcup_{j=1, j \neq i}^{n} H_{M}\left(S_{1}\left(F_{j}\right)\right)
$$

Следовательно,

$$
C_{M}\left(S_{1}\left(F_{i}\right)\right)=M \backslash \bigcup_{j=1, j \neq i}^{n} H_{M}\left(S_{1}\left(F_{j}\right)\right)=\bigcap_{j=1, j \neq i}^{n}\left(M \backslash H_{M}\left(S_{1}\left(F_{j}\right)\right)\right) .
$$

По условию $\left|S_{0}(M)\right|=1$. Поэтому согласно следствию 5 неравенство $\left|S_{0}\left(M_{i}\right)\right|=1$ верно для всех $i \in\{1, \ldots, n\}$. При $i \in\{1, \ldots, n\}$ обозначим через $m_{i}$ единственный элемент множества $S_{0}\left(M_{i}\right)$. Теперь

$$
\begin{aligned}
C_{M}\left(S_{1}\left(F_{i}\right)\right) & =\bigcap_{j=1, j \neq i}^{n}\left(M \backslash\left(M_{1} \times \ldots \times M_{j-1} \times\left(M_{j} \backslash\left\{m_{j}\right\}\right) \times M_{j+1} \times \ldots \times M_{n}\right)\right) \\
& =\bigcap_{j=1, j \neq i}^{n} M_{1} \times \ldots \times M_{j-1} \times\left\{m_{j}\right\} \times M_{j+1} \times \ldots \times M_{n} \\
& =\left\{m_{1}\right\} \times \ldots \times\left\{m_{i-1}\right\} \times M_{i} \times\left\{m_{i+1}\right\} \times \ldots \times\left\{m_{n}\right\}=F_{i} .
\end{aligned}
$$

Лемма доказана. 
Будем называть частично упорядоченное множество $M$ простым, если $|M| \geqslant 2$ и для любой пары частично упорядоченных множеств $P$ и $Q$ из существования изоморфизма $M \cong P \times Q$ следует, что либо $|P|=1$, либо $|Q|=1$.

Лемма 2. Пусть частично упорлдоченное множество $M=M_{1} \times \ldots \times M_{n}$, $\left|S_{0}(M)\right|=1$, а набор множеств $\left\{F_{i}\right\}_{i=1}^{n}$ являетсл каркасом декартова множества наборов $M_{1} \times \ldots \times M_{n}$. Тогда для любого $T \subseteq S_{1}(M)$, для которого множество $C_{M}(T)$ являетсл простым, существует такое $i \in\{1, \ldots, n\}$, ито $T \subseteq S_{1}\left(F_{i}\right)$.

Доказательство. Согласно предложению 6,

$$
S_{1}(M)=\bigcup_{i=1}^{n} S_{1}\left(F_{i}\right),
$$

и $T \subseteq S_{1}(M)$ по условию. Следовательно, если для $i \in\{1, \ldots, n\}$ положить

$$
T_{i}=\left(S_{1}(M) \backslash T\right) \cap S_{1}\left(F_{i}\right)
$$

To

$$
S_{1}(M) \backslash T=\bigcup_{i=1}^{n} T_{i} .
$$

Воспользовавшись предложением 7, получаем, что

$$
\begin{aligned}
& C_{M}(T)=M \backslash H_{M}\left(S_{1}(M) \backslash T\right) \\
& \quad=M \backslash H_{M}\left(\bigcup_{i=1}^{n} T_{i}\right)=M \backslash \bigcup_{i=1}^{n} H_{M}\left(T_{i}\right)=\bigcap_{i=1}^{n}\left(M \backslash H_{M}\left(T_{i}\right)\right) .
\end{aligned}
$$

Так как по определению $T_{i} \subseteq S_{1}\left(F_{i}\right)$ для всех $i \in\{1, \ldots, n\}$, для каждого $i \in\{1, \ldots, n\}$ существует такое множество $P_{i} \subseteq S_{1}\left(M_{i}\right)$, что

$$
T_{i}=\left\{m_{1}\right\} \times \ldots \times\left\{m_{i-1}\right\} \times P_{i} \times\left\{m_{i+1}\right\} \times \ldots \times\left\{m_{n}\right\} .
$$

При $i \in\{1, \ldots, n\}$ положим $Q_{i}=M_{i} \backslash H_{M_{i}}\left(P_{i}\right)$. В этом случае $Q_{i} \neq \varnothing$ для каждого $i \in\{1, \ldots, n\}$, так как $m_{i} \in Q_{i}$. Таким образом,

$$
\begin{aligned}
C_{M}(T) & =\bigcap_{i=1}^{n}\left(M \backslash M_{1} \times \ldots \times M_{i-1} \times H_{M_{i}}\left(P_{i}\right) \times M_{i+1} \times \ldots \times M_{n}\right) \\
& =\bigcap_{i=1}^{n}\left(M_{1} \times \ldots \times M_{i-1} \times Q_{i} \times M_{i+1} \times \ldots \times M_{n}\right)=Q_{1} \times \ldots \times Q_{n} .
\end{aligned}
$$

По условию $C_{M}(T)$ простое. Поэтому существует такое $j \in\{1, \ldots, n\}$, что $Q_{i}=\left\{m_{i}\right\}$ для всех $i \in\{1, \ldots, n\} \backslash\{j\}$. Так как $Q_{i}=M_{i} \backslash H_{M_{i}}\left(P_{i}\right)$, то $H_{M_{i}}\left(P_{i}\right)=M_{i} \backslash\left\{m_{i}\right\}$ для всех $i \in\{1, \ldots, n\} \backslash\{j\}$. Справедливо включение $P_{i} \subseteq S_{1}\left(M_{i}\right)$, следовательно, $P_{i}=S_{1}\left(M_{i}\right)$ при $i \in\{1, \ldots, n\} \backslash\{j\}$, то есть $T_{i}=S_{1}\left(F_{i}\right)$ при $i \in\{1, \ldots, n\} \backslash\{j\}$. А так как

$$
\bigcup_{i=1}^{n} T_{i}=S_{1}(M) \backslash T
$$


To

$$
\bigcup_{i=1, i \neq j}^{n} S_{1}\left(F_{i}\right) \subseteq S_{1}(M) \backslash T .
$$

В итоге, воспользовавшись предложением 6 , получаем, что

$$
T \subseteq S_{1}(M) \backslash \bigcup_{i=1, i \neq j}^{n} S_{1}\left(F_{i}\right)=S_{1}\left(F_{j}\right)
$$

Лемма доказана.

Теорема 1. Пусть частично упорядоченное множество $M=M_{1} \times \ldots \times M_{n}$, $\left|S_{0}(M)\right|=1$, множества $M_{i}$ являютсл простыми при $i \in\{1, \ldots, n\}$, а набор множеств $\left\{F_{i}\right\}_{i=1}^{n}$ является каркасом декартова множества наборов $M_{1} \times \ldots \times M_{n}$. Тогда, если набор множеств $T_{1}, \ldots, T_{k}$ - все махсимальные по включению подмножества $S_{1}(M)$ такие, что множества $C_{M}\left(T_{1}\right), \ldots, C_{M}\left(T_{k}\right)$ простье, то $k=n u$ набор множеств $T_{1}, \ldots, T_{k}$ с точностью до перестановки совпадает $c$ набором множеств $S_{1}\left(F_{1}\right), \ldots, S_{1}\left(F_{n}\right)$.

Доказателъство. По лемме 1 равенство $C_{M}\left(S_{1}\left(F_{i}\right)\right)=F_{i}$ справедливо для каждого $i \in\{1, \ldots, n\}$. Кроме того, $F_{i} \cong M_{i}$ при $i \in\{1, \ldots, n\}$ согласно предложению 5 , и все множества $M_{i}$ являются простыми по условию теоремы. Следовательно, множество $C_{M}\left(S_{1}\left(F_{i}\right)\right)$ является простым для каждого $i \in\{1, \ldots, n\}$.

Так как по условию теоремы множество $C_{M}\left(T_{i}\right)$ является простым для любого $i \in\{1, \ldots, k\}$, по лемме 2 для каждого $i \in\{1, \ldots, k\}$ существует такое $j \in\{1, \ldots, n\}$, что $T_{i} \subseteq S_{1}\left(F_{j}\right)$. Из максимальности $T_{i}$ и простоты $C_{M}\left(S_{1}\left(F_{j}\right)\right)$ получаем, что $T_{i}=S_{1}\left(F_{j}\right)$. Таким образом, набор множеств $T_{1}, \ldots, T_{k}$ является подмножеством набора множеств $S_{1}\left(F_{1}\right), \ldots, S_{1}\left(F_{n}\right)$.

Согласно предложению 5 , справедливо разложение

$$
S_{1}(M)=\bigcup_{i=1}^{n} S_{1}\left(F_{i}\right)
$$

из простоты $F_{i}$ следует неравенство $S_{1}\left(F_{i}\right) \neq \varnothing$ при $i \in\{1, \ldots, n\}$, по ранее доказанному $C_{M}\left(S_{1}\left(F_{i}\right)\right)$ является простым для всех $i \in\{1, \ldots, n\}$, и по условию теоремы набор множеств $T_{1}, \ldots, T_{k}$ является максимальным по включению набором подмножеств множества $S_{1}(M)$ таким, что множества $C_{M}\left(T_{1}\right), \ldots, C_{M}\left(T_{k}\right)$ простые, поэтому набор множеств $T_{1}, \ldots, T_{k}$ с точностью до перестановки совпадает с набором множеств $S_{1}\left(F_{1}\right), \ldots, S_{1}\left(F_{n}\right)$. Теорема доказана.

Пусть для частично упорядоченного множества $M$ верно равенство $\left|S_{0}(M)\right|=1$ и существует покомпонентное разложение

$$
\psi: M \rightarrow M_{1} \times \ldots \times M_{n},
$$

при котором множества $M_{i}$ являются простыми для всех $i \in\{1, \ldots, n\}$. Рассмотрим набор множеств $\left\{F_{i}\right\}_{i=1}^{n}-$ каркас декартова множества наборов $M_{1} \times \ldots \times M_{n}$. Тогда набор множеств $\left\{\psi^{-1}\left(F_{i}\right)\right\}_{i=1}^{n}$ мы будем называть простым каркасом частично упорядоченного множества $M$. 
Теорема 2. Пусть для частично упорядоченного множества $M$ справедливо неравенство $|M| \geqslant 2$ и $\left|S_{0}(M)\right|=1$. Тогда в частично упорядоченном множестве $M$ существует единственный с точностъю до перестановки простой каркас.

Доказательство. Рассмотрим два покомпонентных разложения

$$
\begin{gathered}
\cdot: M \rightarrow M_{1} \times \ldots \times M_{n} \\
\varphi: M \rightarrow N_{1} \times \ldots \times N_{l}
\end{gathered}
$$

в которых все множества $M_{i}$ и $N_{j}$ являются простыми. Пусть $\left\{G_{i}\right\}_{i=1}^{n}-$ простой каркас частично упорядоченного множества $M$ при покомпонентном разложении $\psi$, a $\left\{H_{i}\right\}_{i=1}^{l}-$ простой каркас частично упорядоченно множеств $M$ при покомпонентном разложении $\varphi$. Так как

$$
S_{1}(M) \cong S_{1}\left(M_{1} \times \ldots \times M_{n}\right) \cong S_{1}\left(N_{1} \times \ldots \times N_{l}\right)
$$

и множество $C_{M}(T)$ определяется только отношением сравнимости, утверждение теоремы 1 справедливо и для простых каркасов в $M$. Поскольку для $T \subseteq S_{1}(M)$ множество $C_{M}(T)$ определяется независимо от $\psi$ и $\varphi$, набор $T_{1}, \ldots, T_{k}$ в теореме 1 однозначно определяется по множеству $M$. Следовательно, $k=n=l$ и существует такая перестановка $\pi \in S(n)$, что $S_{1}\left(G_{i}\right)=S_{1}\left(H_{\pi(i)}\right)$ для всех $i \in\{1, \ldots, n\}$.

Тогда по лемме 1

$$
G_{i}=C_{M}\left(S_{1}\left(G_{i}\right)\right)=C_{M}\left(S_{1}\left(H_{\pi(i)}\right)\right)=H_{\pi(i)}
$$

для всех $i \in\{1, \ldots, n\}$.

Теорема доказана.

Пусть $M \cong M_{1}^{k_{1}} \times \ldots \times M_{n}^{k_{n}}$. Тогда декартово произведение $M_{1}^{k_{1}} \times \ldots \times M_{n}^{k_{n}}$ называется разложением частично упорядоченного множества $M$ на простые множители, если все $\left\{M_{i}\right\}_{i=1}^{n}-$ простые попарно неизоморфные множества.

Следствие 6. Пустъ для частично упорядоченного множества $M$ справедливо неравенство $|M| \geqslant 2$ и $\left|S_{0}(M)\right|=1$. Тогда частично упорядоченное множество $M$ раскладывается на простые множстели единственным с точностъю до порядка сомножителей образом.

Обозначим через $\mathrm{Au}(M)$ число автоморфизмов частично упорядоченного множества $M$.

Следствие 7. Пусть для частично упорядоченного множества $M$ справедливо неравенство $|M| \geqslant 2,\left|S_{0}(M)\right|=1$ и дехартово произведение $M_{1}^{k_{1}} \times \ldots \times M_{n}^{k_{n}}$ является разложением частично упорядоченного множества $M$ на простые множители. Tогда

$$
\mathrm{Au}(M)=\prod_{i=1}^{n} \mathrm{Au}\left(M_{i}\right)^{k_{i}} k_{i} !
$$




\section{3. Нижняя оценка сложности сортировки}

При получении нижней оценки сложности сортировки декартовых произведений частично упорядоченного множества мы воспользуемся методом, предложенным в [3]. Вот те идеи из [3], которые были перенесены нами с булева куба на декартовы произведения частично упорядоченно множеств: оценивать снизу сложность более простой задачи сортировки с априорной информацией о двух границах, воспользоваться особой функцией потенциала для вершин дерева сортировки и метками для его ребер, применять леммы 3 и 4 (без изменений).

Пусть $h_{1}, h_{2} \in \mathbf{N}$ и $0 \leqslant h_{1}<h_{2} \leqslant h(M)$. Задачей сортировки частично упорядоченно множеств $M$ с априорной информацией о двух границах $h_{1}$ и $h_{2}$ называется задача сортировки частично упорядоченно множеств $M=\left\{x_{1}, \ldots, x_{m}\right\}$ при наличии предварительной информации о том, что

$$
\begin{cases}l_{M}\left(x_{i}\right)<h_{1} & \text { при } 1 \leqslant i \leqslant k_{1}, \\ h_{1} \leqslant l_{M}\left(x_{i}\right) \leqslant h_{2} & \text { при } k_{1}+1 \leqslant i \leqslant k_{2}, \\ l_{M}\left(x_{i}\right)>h_{2} & \text { при } k_{2}+1 \leqslant i \leqslant m,\end{cases}
$$

где

$$
k_{1}=\sum_{i=0}^{h_{1}-1}\left|S_{i}(M)\right|, \quad k_{2}=\sum_{i=0}^{h_{2}}\left|S_{i}(M)\right| .
$$

Аналогично задаче сортировки частично упорядоченного множества $M$ для задачи сортировки частично упорядоченного множества $M$ с априорной информацией о двух границах $h_{1}$ и $h_{2}$ определяется алгоритм сортировки, его время работы, сложность, вводится́ понятие оптимального алгоритма. Сложность оптимального алгоритма сортировки частично упорядоченного множества $M$ с априорной информацией о двух границах $h_{1}$ и $h_{2}$ мы будем обозначать через $L_{h_{1}, h_{2}}(M)$.

Предложение 8. Пусть дано частично упорлдоченное множество $M$ u $h_{1}, h_{2}$ таxue, ито $h_{1}, h_{2} \in \mathbf{N} u 0 \leqslant h_{1}<h_{2} \leqslant h(M)$. Tогдa $L_{h_{1}, h_{2}}(M) \leqslant L(M)$.

Обозначим через $V$ множество вершин графа диаграммы Хассе частично упорядоченного множества $M$. Будем называть отображение $\theta: M \rightarrow V$ согласованным с последовательностью сравнений $\left\{R\left(x_{p_{i}}, x_{q_{i}}\right)=r_{i}\right\}_{i=1}^{k}$, если для всех $i \in\{1, \ldots, k\}$ при $r_{i}=>$ существует невырожденная ориентированная цепь из вершины $\theta\left(x_{p_{i}}\right)$ в вершину $\theta\left(x_{q_{i}}\right)$, при $r_{i}=<$ существует невырожденная ориентированная цепь из вершины $\theta\left(x_{q_{i}}\right)$ в вершину $\theta\left(x_{p_{i}}\right)$, при $r_{i}=$ ? не существует ориентированных цепей из вершины $\theta\left(x_{p_{i}}\right)$ в вершину $\theta\left(x_{q_{i}}\right)$ и из вершины $\theta\left(x_{q_{i}}\right)$ в вершину $\theta\left(x_{p_{i}}\right)$. Отображение $\theta: M \rightarrow V$, согласованное с последовательностью сравнений

$$
\left\{R\left(x_{p_{i}}, x_{q_{i}}\right)=r_{i}\right\}_{i=1}^{k}
$$

называется единственным с точностью до автоморфизма, если для любого отображения $\theta^{\prime}: M \rightarrow V$, согласованного с последовательностью сравнений

$$
\left\{R\left(x_{p_{i}}, x_{q_{i}}\right)=r_{i}\right\}_{i=1}^{k},
$$

существует такой автоморфизм $\psi: V \rightarrow V$, что $\theta^{\prime}=\psi \theta$.

Назовем деревом сортировки частично упорядоченного множества $(M, R)$ произвольное помеченное корневое ориентированное дерево, каждой внутренней вершине 
$v$ которого приписана пара чисел $\left(p_{v}, q_{v}\right)$, каждому ребру $(u, v)$ которого приписана метка $C(u, v) \in\{<,>, ?\}$ так, что метки на ребрах с общим началом различны, а каждой внешней вершине $v$ приписана сборка $\varphi_{v}$. Деревом сортировки алгоритма сортировки частично упорядоченного множеств $(M, R)$ назовем такое дерево сортировки частично упорядоченного множеств $(M, R)$, что для любой вершины $v$ этого дерева путь $v_{0}, v_{1}, \ldots, v_{k}$ из корня $v_{0}$ дерева сортировки в эту вершину $v=v_{k}$ удовлетворяет следующему условию: после того, как алгоритм сортировки проведет цепочку сравнений $\left\{R\left(x_{p_{v_{i}}}, x_{q_{v_{i}}}\right)=C\left(v_{i}, v_{i+1}\right)\right\}_{i=0}^{k-1}$, он либо будет сравнивать $x_{p_{v}}$ с $x_{q_{v}}$, если вершина $v$ внутренняя (в этом случае предполагается наличие двух или трех исходов сравнимости и для любого из возможных исходов $r$ должно существовать единственное ребро с началом в $v$ и меткой $r$ ), либо получит единственную с точностью до автоморфизма сборку $\varphi_{v}$, согласованную с последовательностью сравнений $\left\{R\left(x_{p_{v_{i}}}, x_{q_{v_{i}}}\right)=C\left(v_{i}, v_{i+1}\right)\right\}_{i=0}^{k-1}$, если вершина $v$ внешняя.

Частично упорядоченное множество $M=\left\{x_{1}, \ldots, x_{m}\right\}$ с отношением частичного порядка $R$ называется согласованным с вершиной $v$ дерева сортировки $T$ алгоритма сортировки частично упорядоченного множества $M$, если на пути $v_{0}, v_{1}, \ldots, v_{k}$ из корня $v_{0}$ дерева $T$ в эту вершину $v=v_{k}$ для любого $i \in\{0,1, \ldots, k-1\}$ верно равенство $C\left(v_{i}, v_{i+1}\right)=R\left(x_{p_{v_{i}}}, x_{q_{v_{i}}}\right)$. На вершинах дерева сортировки $T$ алгоритма сортировки частично упорядоченного множества $M=\left\{x_{1}, \ldots, x_{m}\right\}$ для каждого $i \in\{1, \ldots, m\}$ определим две функции

$$
\alpha_{i}(v)=\max \left\{l_{X}(y): y=g\left(x_{i}\right), g: M \rightarrow X, X \text { согласовано с } v, g \text { - изоморфизм }\right\}
$$

И

$$
\beta_{i}(v)=\min \left\{l_{X}(y): y=g\left(x_{i}\right), g: M \rightarrow X, X \text { согласовано с } v, g \text { - изоморфизм }\right\} .
$$

Назовем функцию

$$
p(v)=\sum_{i=1}^{m}\left(\alpha_{i}(v)-\beta_{i}(v)\right)
$$

потенциалом вершины $v$. Обозначим через $r$ потенциал корня дерева $T$, через $h$ высоту дерева $T$, а через $t$ число его внешних узлов.

Предложение 9. Пусть $v$ - внешняя вершина дерева сортировки $T$ алгоритма сортировки частично упорядоченного множества $M$. Тогда $p(v)=0$.

Предложение 10. Для дерева сортировки Т алгоритма сортировки частично упорлдоченного множества $M$ с априорной информацией о двух границах $h_{1} u h_{2}$ при $h_{1}, h_{2} \in \mathbf{N} u 0<h_{1}<h_{2}<h(M)$ справедливо равенство

$$
r=\left(h_{1}-1\right) k_{1}+\left(h_{2}-h_{1}\right)\left(k_{2}-k_{1}\right)+\left(h(M)-h_{2}-1\right)\left(|M|-k_{2}\right) .
$$

Предложение 11. Пусть в дереве сортировки $T$ алгоритма сортировки частично упорядоченного множества $M=\left\{x_{1}, \ldots, x_{m}\right\}$ вершина $v$ является съном вершины $u$. Тогда при $i \in\{1, \ldots, m\}$ верно, что $\alpha_{i}(v) \leqslant \alpha_{i}(u) u \beta_{i}(v) \geqslant \beta_{i}(u)$.

Следствие 8. Пусть в дереве сортировки $T$ алгоритма сортировки частично упорядоченного множества $M$ вершина $v$ - сън вериины $u$. Тогда $p(v) \leqslant p(u)$.

Назовем ребро $(u, v)$ дерева сортировки $T$ алгоритма сортировки частично упорядоченного множества $M$ проводящим, если $p(u) \neq p(v)$. 
Лемма 3. Пусть вершина и дерева сортировки $T$ алгоритма сортировки частично упорядоченного множества $M$ имеет трех сыновей $v_{1}, v_{2}, v_{3}$. Тогда хотя бы одно из ребер $\left(u, v_{1}\right),\left(u, v_{2}\right),\left(u, v_{3}\right)$ является проводящим.

Доказательство. Не ограничивая общности, будем считать, что $\alpha_{p_{u}}(u) \leqslant \alpha_{q_{u}}(u)$ и $C\left(u, v_{1}\right)=<$. Так как $x_{p_{u}}<x_{q_{u}}$, то и $\alpha_{p_{u}}\left(v_{1}\right)>\alpha_{q_{u}}\left(v_{1}\right)$. Из предложения 11 получаем, что $\alpha_{p_{u}}\left(v_{1}\right) \leqslant \alpha_{p_{u}}(u)$. Таким образом, справедлива цепочка неравенств

$$
\alpha_{q_{u}}\left(v_{1}\right)<\alpha_{p_{u}}\left(v_{1}\right) \leqslant \alpha_{p_{u}}(u) \leqslant \alpha_{q_{u}}(u)
$$

Следовательно, $p\left(v_{1}\right)<p(u)$ и ребро $\left(u, v_{1}\right)$ проводящее.

Лемма доказана.

Лемма 4. Для дерева сортировки Т алгоритма сортировки частично упорядоченного множества $M$ справедлива оценка

$$
t \leqslant\left(\begin{array}{c}
h+r \\
r
\end{array}\right) 2^{h}
$$

Доказателъство. Каждое ребро дерева $T$ пометим буквами из алфавита $\{a, b, c\}$ согласно следующему правилу: если у вершины $u$ есть только два сына $v_{1}$ и $v_{2}$, то ребра $\left(u, v_{1}\right)$ и $\left(u, v_{2}\right)$ метим разными буквами из алфавита $\{a, b\}$, в противном случае у вершины $u$ три сына $v_{1}, v_{2}, v_{3}$ и мы метим ребра $\left(u, v_{1}\right),\left(u, v_{2}\right),\left(u, v_{3}\right)$ разными буквами из алфавита $\{a, b, c\}$ так, чтобы буква $c$ досталась проводящему ребру (возможность такой расстановки меток следует из леммы 3 ). Пусть $w_{1}, \ldots, w_{t}$ - слова, полученные при прочтении реберных меток от корня дерева $T$ к его внешним вершинам. Очевидно, что они различны и длина каждого из них не превосходит $h$. Кроме того, в каждом из этих слов буква $c$ встречается не более $r$ раз. Каждому слову $w_{i}$ произвольным образом припишем справа последовательности букв из алфавита $\{a, b, c\}$ так, чтобы получившиеся слова имели длину $h+r$ и содержали ровно по $r$ букв c. Получившиеся слова различны и их число не превосходит $\left(\begin{array}{c}h+r \\ r\end{array}\right) 2^{h}$. Лемма доказана.

Лемма 5. Для дерева сортировки Т алгоритма сортировки частично упорядоченного множества $M$ с априорной информачией о двух граничах $h_{1} u h_{2}$ справедлива оченка

$$
t \geqslant \frac{k_{1} !\left(k_{2}-k_{1}\right) !\left(|M|-k_{2}\right) !}{\operatorname{Au}(M)}
$$

Доказательство. Число различных корректных входов для задачи сортировки частично упорядоченного множества $M$ с априорной информацией о двух границах $h_{1}$ и $h_{2}$ равно $k_{1} !\left(k_{2}-k_{1}\right) !\left(|M|-k_{2}\right)$ !. Поэтому число неразличимых с точностью до автоморфизма сборок равно

$$
\frac{k_{1} !\left(k_{2}-k_{1}\right) !\left(|M|-k_{2}\right) !}{\operatorname{Au}(M)}
$$

Лемма доказана. 
Следствие 9. Для дерева сортировки $T$ алгоритма сортировки частично упорядоченного мнохсества $M$ с априорной ияформачией о двух граничах $h_{1} u h_{2}$ справедлива оченка

$$
h \geqslant \log _{3} \frac{k_{1} !\left(k_{2}-k_{1}\right) !\left(|M|-k_{2}\right) !}{\mathrm{Au}(M)} .
$$

Теорема 3. Пусть $\left\{M_{n}\right\}$ - бесконечное семейство частично упорядоченябх множсеств такое, ито $\left|M_{n}\right|=m_{n} \rightarrow \infty$ при $n \rightarrow \infty$. Пусть существуют две бесконечнъе последовательности $\left\{h_{1, n}\right\} u\left\{h_{2, n}\right\}$ тахие, что $h_{1, n}, h_{2, n} \in \mathbf{N} u$

$$
0<h_{1, n}<h_{2, n}<h\left(M_{n}\right)
$$

nрu $n \in \mathbf{N}, h_{2, n}-h_{1, n}=o\left(h\left(M_{n}\right)\right)$ npu $n \rightarrow \infty$, a $\partial \Omega \Omega$

$$
k_{1, n}=\sum_{i=0}^{h_{1, n}-1}\left|S_{i}\left(M_{n}\right)\right|, \quad k_{2, n}=\sum_{i=0}^{h_{2, n}}\left|S_{i}\left(M_{n}\right)\right|
$$

справедливо соотношение $k_{2, n}-k_{1, n} \sim m_{n}$ при $n \rightarrow \infty$. Кроме того, пусть

$$
\log \mathrm{Au}\left(M_{n}\right)=o\left(m_{n} \log m_{n}\right), \quad h\left(M_{n}\right)=O\left(\log m_{n}\right)
$$

npu $n \rightarrow \infty$.

Toгda

$$
L_{h_{1, n}, h_{2, n}}\left(M_{n}\right) \gtrsim\left|M_{n}\right| \log _{2}\left|M_{n}\right|
$$

Доказательство. Так как $1 \leqslant k_{1, n}<k_{2, n} \leqslant m_{n}$ и $k_{2, n}-k_{1, n} \sim m_{n}$, то $k_{1, n}=o\left(m_{n}\right)$ и $m_{n}-k_{2, n}=o\left(m_{n}\right)$. Из леммы 5 выводим, что

$$
\begin{aligned}
\log _{2} t & \geqslant \log _{2} k_{1, n} !+\log _{2}\left(k_{2, n}-k_{1, n}\right) !+\log _{2}\left(m_{n}-k_{2, n}\right) !-\log _{2} \mathrm{Au}\left(M_{n}\right) \\
& \gtrsim m_{n} \log _{2} m_{n} .
\end{aligned}
$$

Аналогично, по следствию из леммы 5 получаем оценку

$$
h \geqslant \log _{3} \frac{k_{1, n} !\left(k_{2, n}-k_{1, n}\right) !\left(m_{n}-k_{2, n}\right) !}{\operatorname{Au}\left(M_{n}\right)} \gtrsim m_{n} \log _{3} m_{n} .
$$

Согласно предложению 10 ,

$$
\begin{aligned}
r & =\left(h_{1, n}-1\right) k_{1, n}+\left(h_{2, n}-h_{1, n}\right)\left(k_{2, n}-k_{1, n}\right)+\left(h\left(M_{n}\right)-h_{2, n}-1\right)\left(m_{n}-k_{2, n}\right) \\
& =O\left(\log m_{n}\right) o\left(m_{n}\right)+o\left(h\left(M_{n}\right)\right) O\left(m_{n}\right)+O\left(\log m_{n}\right) o\left(m_{n}\right)=o\left(m_{n} \log m_{n}\right) .
\end{aligned}
$$

Из (2) и (3) следует, что $r=o(h)$. В силу леммы 4 справедлива оценка

$$
\begin{aligned}
\log _{2} t & \leqslant h+\log _{2} \frac{(h+r) !}{h ! r !} \\
& =h+\log _{2} \frac{\sqrt{2 \pi(h+r)}((h+r) / e)^{h+r} e^{\theta_{1} /(12(h+r))}}{\sqrt{2 \pi h}(h / e)^{h} e^{\theta_{2} /(12 h)} \sqrt{2 \pi r}(r / e)^{r} e^{\theta_{3} /(12 r)}}
\end{aligned}
$$


где $0<\theta_{1}<1,0<\theta_{2}<1,0<\theta_{3}<1-$ параметры из формулы Стирлинга. Следовательно,

$$
\begin{aligned}
\log _{2} t \leqslant h & +h \log _{2}\left(1+\frac{r}{h}\right)+r \log _{2}\left(1+\frac{h}{r}\right) \\
& +\log _{2} \sqrt{\frac{h+r}{2 \pi h r}}+\log _{2} e^{\theta_{1} /(12(h+r))-\theta_{2} /(12 h)-\theta_{3} /(12 r)} \\
\leqslant & +h O(r / h)+r o(h / r)+o(1) \sim h .
\end{aligned}
$$

Для дерева сортировки оптимального алгоритма сортировки частично упорядоченного множества $M_{n}$ с априорной информацией о двух границах $h_{1, n}$ и $h_{2, n}$ справедливо равенство $L_{h_{1, n}, h_{2, n}}\left(M_{n}\right)=h$. Воспользуемся (4) и (1) для получения итоговой оценки

$$
L_{h_{1, n}, h_{2, n}}\left(M_{n}\right)=h \gtrsim \log _{2} t \gtrsim m_{n} \log _{2} m_{n} .
$$

Теорема доказана.

Следствие 10. В условиях теоремъ 3 справедлива оченка

$$
L\left(M_{n}\right) \gtrsim\left|M_{n}\right| \log _{2}\left|M_{n}\right| \text {. }
$$

Теорема 4. Пусть для бесконечного семейства частично упорядоченнъх множеств $\left\{K_{i}\right\}$ существует такая постолнная $k$, что $K_{i}$ является простьм, $\left|S_{0}\left(K_{i}\right)\right|=1 u\left|K_{i}\right| \leqslant k$ для всех $i \in \mathbf{N}$. Пусть бесконечное семейство частично упорядоченньх множеств $\left\{M_{n}\right\}$ образовано по правилу $M_{n}=K_{1} \times \ldots \times K_{n}$.

Tогда

$$
L\left(M_{n}\right) \gtrsim\left|M_{n}\right| \log _{2}\left|M_{n}\right| \text {. }
$$

Доказательство. Воспользуемся следующей вероятностной моделью. Пусть $i$-е случайное событие состоит в выборе одного из элементов $x_{i} \in K_{i}$ с вероятностью $1 /\left|K_{i}\right|$, а случайная величина $\xi_{i}=l_{K_{i}}\left(x_{i}\right)$. Полученный таким образом случайный набор $x=\left(x_{1}, \ldots, x_{n}\right)$ является случайным элементом множества $M_{n}$, при этом вероятность появления любого элемента равна $1 /\left|M_{n}\right|$. Согласно предложению 3 , случайная величина $\eta_{n}=\xi_{1}+\ldots+\xi_{n}$ задает номер слоя множества $M_{n}$, в который попал элемент $x$. Воспользуемся неравенством Чебышева

$$
\mathbf{P}\left\{\left|\eta_{n}-\mathbf{M} \eta_{n}\right| \geqslant \tau\right\} \leqslant \frac{\mathbf{D} \eta_{n}}{\tau^{2}}
$$

(см., например, [4]). Подставив $\tau=n^{1 / 2+\varepsilon}$, получаем, что

$$
\mathbf{P}\left\{\left|\eta_{n}-\mathbf{M} \eta_{n}\right| \geqslant n^{1 / 2+\varepsilon}\right\} \leqslant \frac{\mathbf{D} \eta_{n}}{n^{1+2 \varepsilon}} .
$$

Пусть $h_{1, n}=\left[\mathbf{M} \eta_{n}-n^{1 / 2+\varepsilon}\right.$, a $h_{2, n}=\left[\mathbf{M} \eta_{n}+n^{1 / 2+\varepsilon}\right]$. Тогда $h_{2, n}-h_{1, n}=o(n)$ при $\varepsilon<1 / 2$. А так как $h\left(M_{n}\right)=\Omega(n)=\Omega\left(\log \left|M_{n}\right|\right)$ из-за ограниченности $\left\{\left|K_{i}\right|\right\}$, то $h_{2, n}-h_{1, n}=o\left(h\left(M_{n}\right)\right)$. Аналогично $\mathbf{D} \eta_{n}=O(n)$. Следовательно, $\mathbf{D} \eta_{n} / n^{1+2 \varepsilon}=$ $o(1)$. Поэтому $\mathbf{P}\left\{\left|\eta_{n}-\mathbf{M} \eta_{n}\right|<n^{1 / 2+\varepsilon}\right\} \rightarrow 1$ при $n \rightarrow \infty$. Но к этой вероятности сходится отношение $\left(k_{2, n}-k_{1, n}\right) /\left|M_{n}\right|$. Поэтому $k_{2, n}-k_{1, n} \sim\left|M_{n}\right|$. По следствию 7 из теоремы 2 получаем оценку

$$
\log _{2} \mathrm{Au}\left(M_{n}\right) \leqslant \log _{2}\left(n ! \prod_{i=1}^{n} \mathrm{Au}\left(K_{i}\right)\right) \leqslant n \log _{2} n+n k \log _{2} k=o\left(\left|M_{n}\right| \log \left|M_{n}\right|\right) .
$$


Таким образом, для семейства частично упорядоченных множеств $\left\{M_{n}\right\}$ выполняются условия следствия 9 из теоремы 3.

Теорема доказана.

\section{4. Верхняя оценка сложности сортировки}

Рассмотрим частично упорядоченное множество $M$, у которого $\left|S_{0}(M)\right|=1$. Обозначим $U_{l}(M)$ множество элементов из $M$, каждый из которых меньше не более $l$ элементов из $S_{1}(M)$.

Предложение 12. Для частично упорядоченно множеств $M$ c $\left|S_{0}(M)\right|=1$ из неравенства $i \leqslant j$ следует вложение $U_{i}(M) \subseteq U_{j}(M)$.

Лемма 6. Пусть $M$ - частично упорядоченное множество, для которого $|M| \geqslant 2$ $u\left|S_{0}(M)\right|=1$. Пусть $M \cong M_{1} \times \ldots \times M_{n}$ u существует тахал константа $k$, что $\left|M_{i}\right| \leqslant k$ для всех $i \in\{1, \ldots, n\}$. Тогда при $l \in\{1, \ldots, n\}$ справедлива оченка

$$
\left|U_{l}(M)\right| \leqslant \frac{k^{l}}{l !} n^{l}
$$

Доказательство. Рассмотрим произвольное покомпонентное разложение

$$
\psi: M \rightarrow M_{1} \times \ldots \times M_{n}
$$

Множество $U_{l}(M)$ целиком содержится в множестве элементов $\{x\}$ частично упорядоченного множества $M$, среди компонент $x_{i}$ образов $\psi(x)=\left(x_{1}, \ldots, x_{n}\right)$ которых имеется не более $l$ элементов, отличных от максимального элемента соответствующего множества $M_{i}$. Поэтому

$$
\left|U_{l}(M)\right| \leqslant\left(\begin{array}{l}
n \\
l
\end{array}\right) k^{l} \leqslant \frac{n^{l}}{l !} k^{l} .
$$

Лемма доказана.

Лемма 7. Для частично упорядоченного множества $M$ со свойством $\left|S_{0}(M)\right|=1$ и множества $T \subseteq S_{1}(M)$ справедливо равенство

$$
C_{M}(T)=\left\{x \in M: \forall y \in S_{1}(M)((y \geqslant x) \Rightarrow(y \in T))\right\} .
$$

Доказательство. По определению $C_{M}(T)=M \backslash H_{M}\left(S_{1}(M) \backslash T\right)$. Следовательно, утверждение леммы справедливо тогда и только тогда, когда

$$
H_{M}\left(S_{1}(M) \backslash T\right)=M \backslash\left\{x \in M: \forall y \in S_{1}(M)((y \geqslant x) \Rightarrow(y \in T))\right\}
$$

Действительно,

$$
\begin{aligned}
& M \backslash\left\{x \in M: \forall y \in S_{1}(M)((y \geqslant x) \Rightarrow(y \in T))\right\} \\
& =\left\{x \in M: \exists y \in S_{1}(M)\left((y \geqslant x) \wedge\left(y \in S_{1}(M) \backslash T\right)\right)\right\} \\
& =\left\{x \in M: \exists y \in\left(S_{1}(M) \backslash T\right)(y \geqslant x)\right\}=H_{M}\left(S_{1}(M) \backslash T\right) .
\end{aligned}
$$

Лемма доказана. 
Следствие 11. Для частично упорядоченного множества $M$ c $\left|S_{0}(M)\right|=1$ и множества $T \subseteq S_{1}(M)$ справедливо включение $C_{M}(T) \subseteq U_{|T|}(M)$.

Теорема 5. Пусть для бесконечного семейства частично упорядоченных множеств $\left\{K_{i}\right\}$ существует такая постоянная $k$, что $K_{i}$ является простым, $\left|S_{0}\left(K_{i}\right)\right|=1$ u $\left|K_{i}\right| \leqslant k$ для всех $i \in \mathbf{N}$, и пусть $\left\{M_{n}\right\}$ - бесконечное семейство частично упорядоченных множеств, образованных по правилу

$$
M_{n}=K_{1} \times \ldots \times K_{n} .
$$

Toгda

$$
L\left(M_{n}\right) \lesssim\left(\left|K_{1}\right|+\ldots+\left|K_{n}\right|-n\right)\left|M_{n}\right|
$$

Доказательство. Зафиксируем $n \in \mathrm{N}$. При $n=1$ утверждение теоремы верно. Далее предполагаем $n \geqslant 2$. Алгоритм сортировки частично упорядоченного множества $M_{n}$ разобьем на пять шагов.

1. Поиск единственного элемента $S_{0}\left(M_{n}\right)$. Пусть $\left|M_{n}\right|=m_{n}, M_{n}=\left\{x_{1}, \ldots, x_{m_{n}}\right\}$. Рассмотрим следующий процесс: $y_{1}=x_{1}$, а при $i \in\left\{2, \ldots, m_{n}\right\}$, если $x_{i}>y_{i-1}$, то $y_{i}=x_{i}$, в противном случае $y_{i}=y_{i-1}$. При таком построении $y_{m_{n}} \in \max \left(M_{n}\right)$. Таким образом, единственный элемент множества $S_{0}\left(M_{m}\right)$ можно найти за не более чем $m_{n}$ шагов.

2. Вычерпывание слоя $S_{1}\left(M_{n}\right)$. Пусть $s=\left|S_{1}\left(M_{n}\right)\right|$, а $D_{0}=M_{n} \backslash S_{0}\left(M_{n}\right)$. Рассмотрим следующий процесс:

$$
d_{i} \in \max \left(D_{i-1}\right), \quad D_{i}=D_{i-1} \backslash\left\{x \in D_{i-1}: x \leqslant d_{i}\right\}, \quad \in\{1, \ldots, s\} .
$$

Здесь $d_{i}-$ произвольный элемент $\max \left(D_{i-1}\right)$, получаемый по алгоритму шага 1 . По построению, получившееся множество $\left\{d_{i}\right\}_{i=1}^{s}$ совпадает с $S_{1}\left(M_{n}\right)$. Таким образом, сложность вычерпывания слоя $S_{1}\left(M_{n}\right)$ равна $2 \sum_{i=1}^{s}\left|D_{i-1}\right|$. Теперь оценим величину $\left|D_{i}\right|$ для $i \in\{0,1, \ldots, s-1\}$.

По определению

$$
D_{i}=D_{i-1} \backslash\left\{x \in D_{i-1}: x \leqslant d_{i}\right\} .
$$

По построению $S_{0}\left(M_{n}\right) \cap D_{i}=\varnothing$ при $i \in\{0,1, \ldots, s-1\}$. Следовательно, так как $d_{i} \in S_{1}\left(M_{n}\right)$, множество $D_{i-1}$ не содержит элементов, больших $d_{i}$, и поэтому

$$
D_{i}=\left\{x \in D_{i-1}: x ? d_{i}\right\}
$$

По индукции получаем, что

$$
D_{i}=\left\{x \in M_{n} \backslash S_{0}\left(M_{n}\right): x ? d_{1}, \ldots, x ? d_{i}\right\}
$$

Возьмем произвольное покомпонентное разложение

$$
\psi: M_{n} \rightarrow K_{1} \times \ldots \times K_{n}
$$

Так как все $d_{j} \in S_{1}\left(M_{n}\right)$, для образа $\psi\left(d_{j}\right)=\left(d_{j, 1}, \ldots, d_{j, n}\right)$ существует единственный индекс $l_{j} \in\{1, \ldots, n\}$ такой, что $d_{j, l_{j}} \in S_{1}\left(K_{l_{j}}\right)$ и $d_{j, r} \in S_{0}\left(K_{r}\right)$ для всех $r \in\{1, \ldots, n\} \backslash\left\{l_{j}\right\}$. Следовательно, для любого $x \in M_{n} \backslash S_{0}\left(M_{n}\right)$ для образа 
$\psi(x)=\left(x_{1}, \ldots, x_{n}\right)$ отношение $\left(x_{1}, \ldots, x_{n}\right) ?\left(d_{j, 1}, \ldots, d_{j, n}\right)$ справедливо тогда и только тогда, когда либо $x_{l_{j}} ? d_{j, l_{j}}$, либо $x_{l_{j}}>d_{j, l_{j}}$. Таким образом,

$$
\begin{aligned}
D_{i}=\left\{x=\left(x_{1}, \ldots, x_{n}\right) \in M_{n} \backslash S_{0}\left(M_{n}\right):\left(\left(x_{l_{1}} ? d_{1, l_{1}}\right)\right.\right. & \left.\vee\left(x_{l_{1}}>d_{1, l_{1}}\right)\right) \wedge \ldots \\
& \left.\wedge\left(\left(x_{l_{i}} ? d_{i, l_{i}}\right) \vee\left(x_{l_{i}}>d_{i, l_{i}}\right)\right)\right\} .
\end{aligned}
$$

Пусть

$$
\begin{aligned}
D_{i}^{\prime}=\left\{x=\left(x_{1}, \ldots, x_{n}\right) \in M_{n}:\left(( x _ { l _ { 1 } } ? d _ { 1 , l _ { 1 } } ) \vee \left(x_{l_{1}}>\right.\right.\right. & \left.\left.d_{1, l_{1}}\right)\right) \wedge \ldots \\
& \left.\wedge\left(\left(x_{l_{i}} ? d_{i, l_{i}}\right) \vee\left(x_{l_{i}}>d_{i, l_{i}}\right)\right)\right\} .
\end{aligned}
$$

Тогда $D_{i}^{\prime}=D_{i} \cup S_{0}\left(M_{n}\right)$ и, соответственно, $\left|D_{i}^{\prime}\right|=\left|D_{i}\right|+1$. Так как все элементы $d_{i}$ принадлежат $S_{1}\left(M_{n}\right)$ и различны между собой, равенство $l_{i}=l_{j}$ влечет неравенство $d_{i, l_{2}} \neq d_{j, l_{j}}$. Таким образом, при переходе от множества $D_{i-1}^{\prime}$ к множеству $D_{i}^{\prime}$ мы запрещаем хотя бы одно значение (в том числе и $d_{i, l_{i}}$ ) по одной из координат (в данном случае по $l_{i}$ ) покомпонентного разложения элементов множества $M_{n}$. А так как по условию $\left|K_{j}\right| \leqslant k$ для всех $j \in\{1, \ldots, n\}$, мы запретим не менее $1 / k$ части элементов множества $D_{i-1}^{\prime}$. Поэтому,

$$
\frac{\left|D_{i}^{\prime}\right|}{\left|D_{i-1}^{\prime}\right|} \leqslant \frac{k-1}{k}
$$

Следовательно,

$$
\frac{1}{\left|D_{i}^{\prime}\right|}>\frac{1}{\left|D_{i-1}^{\prime}\right|}
$$

и

$$
\frac{\left|D_{i}\right|}{\left|D_{i-1}\right|}=\frac{\left|D_{i}^{\prime}\right|-1}{\left|D_{i-1}^{\prime}\right|-1}=\frac{\left|D_{i}^{\prime}\right|\left(1-1 /\left|D_{i}^{\prime}\right|\right)}{\left|D_{i-1}^{\prime}\right|\left(1-1 / \mid D_{i-1}^{\prime}\right)}<\frac{k-1}{k} \text {. }
$$

В итоге получаем, что оценка сложности шага 2 равна

$$
2 \sum_{i=1}^{s}\left|D_{i-1}\right|<2\left|D_{0}\right| \sum_{i=0}^{\infty}((k-1) / k)^{i}<2 k m_{n}
$$

3. Подготовка к извлечению каркаса. Пусть

$$
l=\max \left\{\left|S_{1}(K)\right|: K \in\left\{K_{i}\right\}\right\} .
$$

Этот максимум существует в силу ограниченности множества $\left\{\left|K_{i}\right|\right\}$. Сравнивая все элементы $S_{1}\left(M_{n}\right)$ со всеми элементами $M_{n}$, найдем множество $U_{l}\left(M_{n}\right)$. На это нам потребуется не более $\left|S_{1}\left(M_{n}\right)\right| m_{n}$ сравнений. Это и будет сложностью шага 3.

4. Извлечение каркаса. Рассмотрим произвольное покомпонентное разложение

$$
\psi: M_{n} \rightarrow K_{1} \times \ldots \times K_{n} .
$$

Пусть набор множеств $\left\{F_{i}\right\}_{i=1}^{n}-$ каркас декартова множества наборов $K_{1} \times \ldots \times K_{n}$. Положим $G_{i}=\psi^{-1}\left(F_{i}\right)$ при $i \in\{1, \ldots, n\}$. Тогда набор множеств $\left\{G_{i}\right\}_{i=1}^{n}$ по определению будет простым каркасом частично упорядоченного множества $M_{n}$. Согласно 
теореме 2, этот набор будет единственным с точностью до перестановки. Используя лемму 1 , получаем, что

$$
F_{i}=C_{\psi\left(M_{n}\right)}\left(S_{1}\left(\psi\left(G_{i}\right)\right)\right) .
$$

Тогда включение $F_{i} \subseteq \psi\left(U_{l}\left(M_{n}\right)\right)$ при $i \in\{1, \ldots, n\}$ вытекает из следствия 11 леммы 7 и предложения 12. Следовательно, простой каркас $\left\{G_{i}\right\}_{i=1}^{n}$ целиком содержится в множестве $U_{l}\left(M_{n}\right)$. Попарно сравним между собой все элементы множества $U_{l}\left(M_{n}\right)$. На это потребуется не более $\left|U_{l}\left(M_{n}\right)\right|^{2} / 2$ сравнений. Покажем, что произведенных к данному моменту операций достаточно для выявления простого каркаса $\left\{G_{i}\right\}_{i=1}^{n}$.

Для начала покажем, что этих операций достаточно для определения простоты множества $C_{M_{n}}(T)$ для любого $T \subseteq S_{1}\left(M_{n}\right)$. Так как $M_{n} \cong K_{1} \times \ldots \times K_{n}$ и множество $C_{M_{n}}(T)$ при $T \subseteq S_{1}\left(M_{n}\right)$ определяется только отношением сравнимости, утверждение леммы 2 справедливо и для простых каркасов в $M_{n}$. Теперь по лемме 2 для $T \subseteq S_{1}\left(M_{n}\right)$ из простоты множества $C_{M_{n}}(T)$ следует существование такого $i \in\{1, \ldots, n\}$, что $T \subseteq S_{1}\left(G_{i}\right)$. А так как для любого $i \in\{1, \ldots, n\}$ существует такое $j \in\{1, \ldots, n\}$, что $G_{i} \cong K_{j}$, то $|T| \leqslant l$. Таким образом, воспользовавшись следствием 11 леммы 7 и предложением 12 , получаем, что если множество $C_{M_{n}}(T)$ простое, то $|T| \leqslant l$ и $C_{M_{n}}(T) \subseteq U_{l}\left(M_{n}\right)$. В итоге получаем следующий алгоритм распознавания простоты множества $C_{M_{n}}(T)$ для любого $T \subseteq S_{1}\left(M_{n}\right)$ : если $|T|>l$, то множество $C_{M_{n}}(T)$ заведомо не простое, в противном случае воспользуемся результатами операций шага 3 для построения множества $C_{M_{n}}(T)$ и результатами попарных сравнений элементов множества $U_{l}\left(M_{n}\right)$ для определения простоты множества $C_{M_{n}}(T)$.

Так как $M_{n} \cong K_{1} \times \ldots \times K_{n}$ и множество $C_{M_{n}}(T)$ при $T \subseteq S_{1}\left(M_{n}\right)$ определяется только отношением сравнимости, утверждения леммы 1 и теоремы 1 справедливы и для простых каркасов в $M_{n}$. Согласно теореме 1 , если набор множеств $T_{1}, \ldots, T_{k}$ - все максимальные по включению подмножества $S_{1}\left(M_{n}\right)$ такие, что множества $C_{M_{n}}\left(T_{1}\right), \ldots, C_{M_{n}}\left(T_{k}\right)$ простые, то $k=n$ и набор множеств $T_{1}, \ldots, T_{k}$ с точностью до перестановки совпадает с набором множеств $S_{1}\left(G_{1}\right), \ldots, S_{1}\left(G_{n}\right)$. Для выявление набора множеств $T_{1}, \ldots, T_{n}$ воспользуемся приведенным выше алгоритмом распознавания простоты множества $C_{M_{n}}(T)$ при $T \subseteq S_{1}\left(M_{n}\right)$. Обозначим $H_{i}=C_{M_{n}}\left(T_{i}\right)$ при $i \in\{1, \ldots, n\}$. Согласно лемме 1 , набор множеств $\left\{H_{i}\right\}_{i=1}^{n}$ является одной из перестановок простого каркаса множества $M_{n}$.

5. Развешивание осталъных элементов. По определению простого каркаса существует перестановка $\pi \in S(n)$, при которой для всех $i \in\{1, \ldots, n\}$ существует изоморфизм $\phi_{i}: H_{i} \rightarrow K_{\pi(i)}$. Для каждого $x \in M_{n}$ определим элементы его покомпонентного разложения $\psi(x)=\left(x_{1}, \ldots, x_{i 2}\right)$ согласно правилу

$$
x_{i}=\min \left\{t: t=\phi_{i}(y), y \in H_{i}, y \geqslant x\right\} .
$$

Так как $S_{0}\left(M_{n}\right) \subset H_{i}$ для всех $i \in\{1, \ldots, n\}$, а сравнения всех элементов с элементами $S_{1}\left(M_{n}\right)$ были произведены еще на шаге 3 , на определение всех компонент покомпонентного разложения любого элемент $x \in M_{n}$ будет истрачено не более

$$
\sum_{i=1}^{n}\left(\left|K_{i}\right|-\left|S_{0}\left(K_{i}\right)\right|-\left|S_{1}\left(K_{i}\right)\right|\right)
$$

сравнений. На все $x$ из $M_{n}$ будет истрачено не более

$$
\left(\left|K_{1}\right|+\ldots+\left|K_{n}\right|-n-\left|S_{1}\left(M_{n}\right)\right|\right) m_{n}
$$


сравнений. Это и будет сложностью шага 5.

Согласно предложению 1 , для решения задачи сортировки частично упорядоченного множества $M$ остается для всех $i \in\{1, \ldots, n\}$ задать сборки $\varphi_{i}: K_{i} \rightarrow V_{i}$, где $V_{i}$ - множество вершин диаграммы Хассе частично упорядоченного множества $K_{i}$. Это можно сделать перебором, исходя из операций шага 3.

Алгоритм построен. Оценим его сложность при $n \geqslant l$ и $n \rightarrow \infty$. Ограничение $n \geqslant l$ позволяет применить лемму 6 для оценки сложности шага 3 и получить верхнюю оценку

$$
\begin{aligned}
L\left(M_{n}\right) \leqslant & m_{n}+2 k m_{n}+\left|S_{1}\left(M_{n}\right)\right| m_{n}+\frac{1}{2}\left(\frac{k^{l}}{l !} n^{l}\right)^{2} \\
& +\left(\left|K_{1}\right|+\ldots+\left|K_{n}\right|-n-\left|S_{1}\left(M_{n}\right)\right|\right) m_{n} \\
= & \left(\left|K_{1}\right|+\ldots+\left|K_{n}\right|-n+2 k+1\right) m_{n}+o\left(m_{n}\right) .
\end{aligned}
$$

Теорема доказана.

Следствие 12. В условиях теоремь 5 справедлива оченка

$$
L\left(M_{n}\right) \asymp\left|M_{n}\right| \log \left|M_{n}\right| .
$$

Пример 1. Обозначим через $B$ частично упорядоченное множество мощности 2, один из элементов которого больше другого. Тогда при $n \in \mathbf{N}$ частично упорядоченное множество $B^{n}$ представляет собой классический $n$-мерный куб. Так как множество $B$ является простым и $S_{0}(B)=1$, к частично упорядоченному множеств $B^{n}$ применима теорема 4. Поэтому

$$
L\left(B^{n}\right) \gtrsim\left|B^{n}\right| \log _{2}\left|B_{n}\right|=n 2^{n} .
$$

Аналогично, согласно теореме 5 справедлива оценка

$$
L\left(B^{n}\right) \lesssim(2 n-n) 2^{n}=n 2^{n} .
$$

Следовательно,

$$
L\left(B^{n}\right) \sim n 2^{n} .
$$

Остается заметить, что нижняя оценка для $L\left(B^{n}\right)$ была впервые получена в [3], а верхняя в [5].

\section{Список литературы}

1. Ахо А. В., Хопкрофт Дж., Ульман Дж., Построение и анализ въчислителънъх алгоритмов. Мир, Москва, 1979.

2. Кнут Д., Искусство програм.иирования для ЭВМ. Сортировка и поиск, т. 3. Мир, Москва, 1978.

3. Морозенко В. В., О сложности сортировки булевой алгебры. Дискретная математиха (1991) 3, №1, 42-47.

4. Боровков А. А., Теория вероятностей. Наука, Москва, 1986.

5. Faigle U., Turan G., Sorting and recognition problems for ordered sets. SIAM J. Comput. (1988) 17, №1, 100-113. 